\title{
Ultrasound fetal biometry in third trimester in urban Nepalese population
}

\author{
Bhuwan Kayastha', Shahjan Raj Giri', Bibek Gurung', Om Biju Panta' \\ ${ }^{1}$ Department of Radiology and Imaging, Nepal Mediciti Hospital, Bhaisepati, Lalitpur, Nepal
}

\begin{abstract}
INTRODUCTION

Fetal biometry is an important component of fetal growth surveillance and can detect small for gestation age fetuses. However, we use growth curves from studies done in other high-income countries and do not have a standard of our own. This study aims to study the deviation of biometry and expected fetal weight from these curves in pregnancy with normal birth weight term deliveries.
\end{abstract}

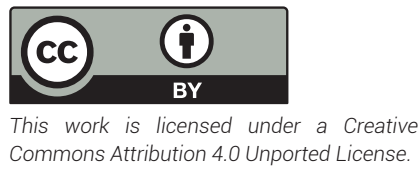

\section{METHODS}

The study was a retrospective hospital record-based study including term delivery from 2018 May to 2020 January. All lowrisk patients visiting ultrasound OPD in our hospital in 3rd trimester were retrieved and included in the study. Patient with Iow AFI, major fetal anomaly, abnormal fetal heart rate, preterm delivery, birth weight less than 2500 gm (low birth weight), intrauterine fetal demise, preeclampsia or other complications were excluded from the study. Fetal biometry and expected fetal weight were evaluated. Data was entered in predesigned performa and analysis was performed with IBM SPSS 20.0.

\section{RESULTS}

A total of 590 ultrasound examinations done in 372 patients were included in the study. Mean age of the patients was $30.78+/-3.98$ years, median age was 30 years (IQ range-5). Approximately 316(53.6\%) patients were under 30 years of age, 198(33.6\%) patients were between 30-35 and 76(12.9\%) patients were above 35 years. Median gestational age at birth was 39 weeks and median birth weight was 3120 grams. Median and percentile for various biometry was calculated. EFW was below 50th percentile in 424(71.9\%) according to WHO chart. The median percentile for EFW was 30.6 (IQ-33.6).

\section{CONCLUSION}

Expected fetal weight in Nepalese population is smaller than that predicted by WHO chart. Development of country specific fetal growth curve is recommended for accurate fetal growth surveillance.

\section{KEY WORD}

Ultrasound, Expected fetal weight, Fetal growth surveillance, Fetal growth chart

\section{INTRODUCTION}

Monitoring and surveillance of fetal size and growth is an essential component of prenatal care. Fetal biometric parameters commonly used in ultrasound assessment of fetal size are biparietal diameter, head circumference, abdominal circumference and femur diaphysis length. Expected fetal weight is calculated from these measurements using various formulas, one commonly used formula is Hadlock 2.(1)(2) Comparing the biometric measurements and expected fetal weight with age specific growth chart helps to identify small, appropriate or large for gestational age fetuses. Accurate gestation age is most essential to assess fetal size and growth which should be calculated from first trimester ultrasound. (2)
However, most charts commonly in practice are developed from studies in European countries. The variation in size due to regional, cultural and ethnical difference cannot be accounted, which might lead to difficulty in differentiation of constitutional variation from pathological changes. World health organization has attempted to resolve this problem by developing a growth chart from multinational study. ${ }^{(3)}$ The purpose of this study is to study the deviation of biometry and expected fetal weight from these curves in pregnancy with normal birth weight term deliveries.

\footnotetext{
*Corresponding Author I

Dr. Bhuwan Kayastha | Department of Radiology and

Imaging, Nepal Mediciti Hospital, Bhaisepati, Lalitpur, Nepal Email: bhuwan.kayastha@nepalmediciti.com
} 


\section{METHODS}

The study was a retrospective observational study conducted in the department of radiology and imaging. Our patient population was all term deliveries (normal delivery, emergency or elective cesarian section) in our hospital from 2018 May to 2020 January. Retrospective study of ultrasound images on PACS was done. All low-risk patients visiting ultrasound OPD in our hospital in 3rd trimester were retrieved and included in the study. Obstetric ultrasound was conducted in Siemens ACUSON S 3000 USG machine. Biparietal diameter (BPD), Head circumference (HC), Abdominal circumference (AC) and Femoral length (FL) were measured by two observers with 2 years and 4 years' experience in Obstetric ultrasound. Estimated fetal weight (EFW) were calculated using Hadlock 3 formula. Amniotic fluid index and fetal heart rate were also measured. Patient with low AFI, major fetal anomaly, abnormal fetal heart rate, preterm delivery, birth weight less than 2500 gm (low birth weight), intrauterine fetal demise, preeclampsia or other complications were excluded from the study. Gestational age at ultrasound was calculated from last menstrual period. Median, 5th, 25th, 50th, 75th and 95th percentile for BPD, HC, AC, FL and EFW was calculated for various gestational age. We calculated percentile of expected fetal weight for the corresponding gestational age using WHO growth chart. Statistical analysis was done using IBM SPSS version 26.

\section{RESULTS}

A total of 590 ultrasound examinations done in 372 patients were included in the study. 200 patients had a single scan, 126 patients had 2 scans and 46 patients had 3 scans between 30-40 weeks. Mean age of the patients was $30.78+/-3.98$ years, median age was 30 years (IO range-5). Approximately 316(53.6\%) patients were under 30 years of age, 198(33.6\%) patients were between 3035 and $76(12.9 \%)$ patients were above 35 years. Median gestational age at birth was 39 weeks and median birth weight was 3120 grams.

Median, 5th, 25th, 75th and 95th percentile for BPD, HC, AC, FL and EFW is detailed in table 1-5 and Figure 1 to 5.

Percentile for EFW according to WHO chart was calculated for our population. Mean percentile for EFW was 36.15+/24.11. Median percentile for EFW was 30.6 with IQ range of 33.6, maximum and minimum were 90.6 and 2.5 percentiles. Approximately $424(71.9 \%)$ of scans had EFW below 50th percentile and approximately 25(4.2\%) of scans had EFW below 5 percentiles according to $\mathrm{WHO}$ chart.

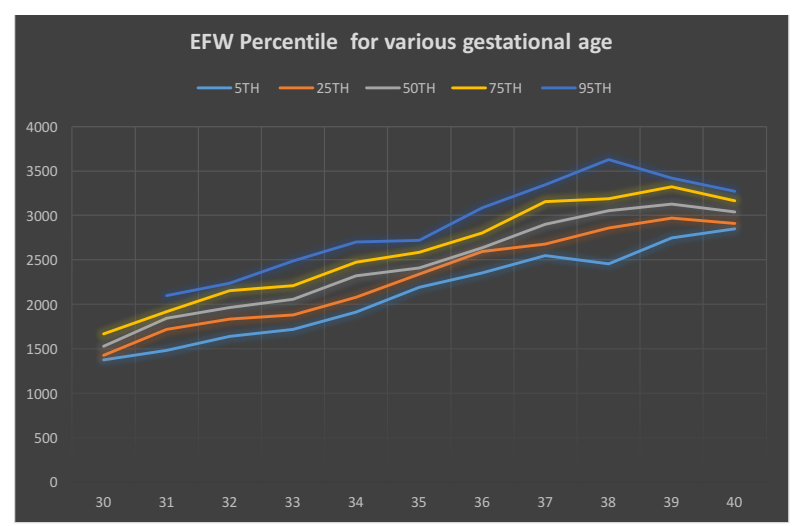

Figure 1. EFW percentile for various gestational age

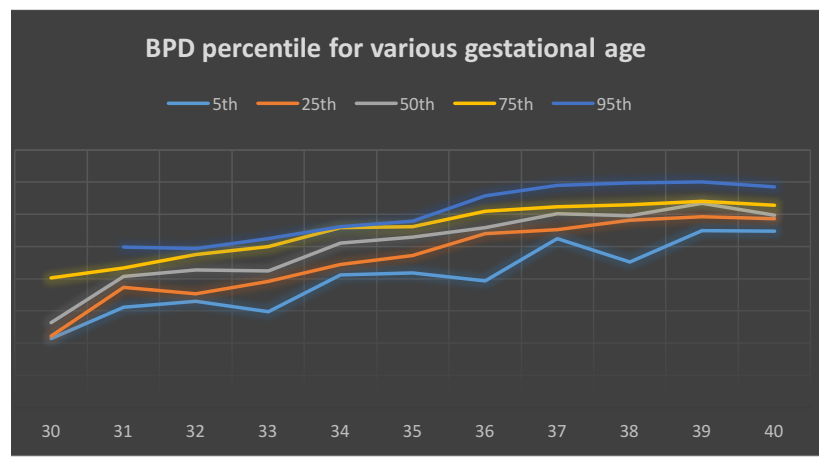

Figure 2. BPD percentile for various gestational age.

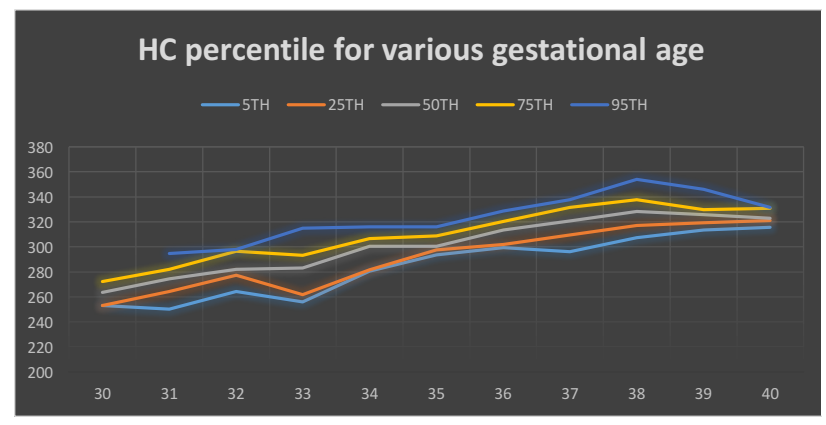

Figure 3. HC percentile for various gestational age.

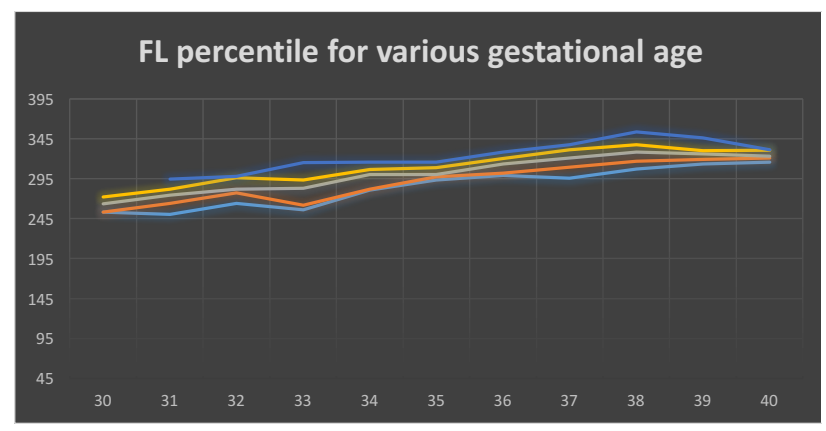

Figure 4. FL percentile for various gestational age 


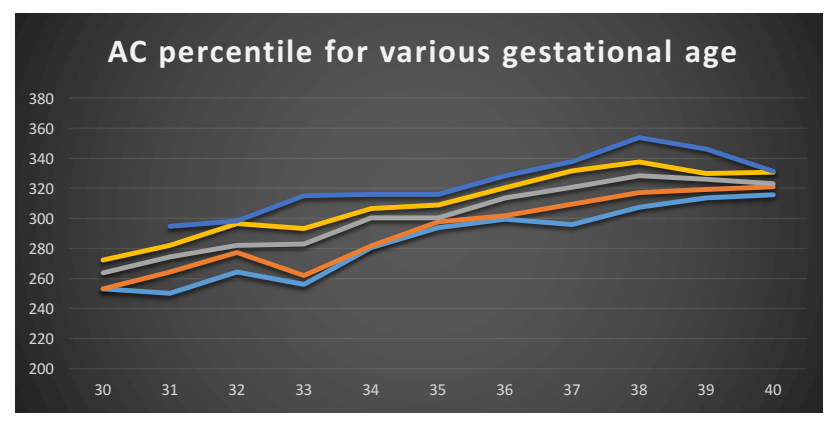

Figure 5. AC percentile for various gestational age

Table 1: EFW percentile chart for various gestational age

\begin{tabular}{|l|l|l|l|l|l|}
\hline WEEKS (N) & \multicolumn{1}{|c|}{5 TH } & 25TH & 50TH & 75TH & 95TH \\
\hline 30 & 1377 & 1425 & 1526 & 1667 & \\
\hline 31 & 1481 & 1716 & 1842 & 1919 & 2100 \\
\hline 32 & 1639 & 1835 & 1965 & 2154 & 2237 \\
\hline 33 & 1718 & 1882 & 2055 & 2207 & 2488 \\
\hline 34 & 1913 & 2081 & 2322 & 2474 & 2699 \\
\hline 35 & 2190 & 2339 & 2410 & 2583 & 2717 \\
\hline 36 & 2351 & 2593 & 2634 & 2804 & 3085 \\
\hline 37 & 2547 & 2679 & 2901 & 3155 & 3347 \\
\hline 38 & 2457 & 2856 & 3052 & 3187 & 3629 \\
\hline 39 & 2745 & 2969 & 3129 & 3323 & 3418 \\
\hline 40 & 2851 & 2909 & 3037 & 3165 & 3271 \\
\hline
\end{tabular}

Table 2. HC percentile chart for various gestational age

\begin{tabular}{|l|l|l|l|l|l|}
\hline \multicolumn{1}{|c|}{ WEEKS } & \multicolumn{1}{c|}{ 5TH } & \multicolumn{1}{c|}{ 25TH } & \multicolumn{1}{c|}{ 50TH } & \multicolumn{1}{c|}{ 75TH } & \multicolumn{1}{c|}{ 95TH } \\
\hline 30 & 268.2 & 269.9 & 271.6 & 285.1 & \\
\hline 31 & 274.4 & 283.7 & 288.4 & 294.5 & 301 \\
\hline 32 & 281.4 & 291.1 & 298.5 & 309.3 & 316.8 \\
\hline 33 & 279.4 & 299.5 & 303.6 & 308.3 & 312.2 \\
\hline 34 & 302.4 & 303.3 & 307.3 & 312.2 & 313.7 \\
\hline 35 & 302.1 & 308.2 & 311.3 & 318.6 & 324.1 \\
\hline 36 & 301.8 & 312.4 & 318 & 325.8 & 332.4 \\
\hline 37 & 304.1 & 320.9 & 326.2 & 331.8 & 338.4 \\
\hline 38 & 304.8 & 323.4 & 326.3 & 332.7 & 339.3 \\
\hline 39 & 311.8 & 319 & 325.8 & 331.1 & 341 \\
\hline 40 & 309.2 & 311.3 & 320.8 & 328.3 & 328.8 \\
\hline
\end{tabular}

Table 3. BPD percentile chart for various gestational age

\begin{tabular}{|l|l|l|l|l|l|}
\hline \multicolumn{1}{|c|}{ WEEKS (N) } & \multicolumn{1}{|c|}{ 5TH } & \multicolumn{1}{c|}{ 25TH } & 50TH & \multicolumn{1}{|c|}{ 75TH } & \multicolumn{1}{c|}{ 95TH } \\
\hline $30(13)$ & 70.7 & 71.1 & 73.2 & 80.1 & \\
\hline $31(45)$ & 75.6 & 78.7 & 80.4 & 81.7 & 84.9 \\
\hline $32(50)$ & 76.5 & 77.7 & 81.4 & 83.8 & 84.7 \\
\hline $33(58)$ & 74.9 & 79.6 & 81.2 & 85.0 & 86.2 \\
\hline $34(35)$ & 80.6 & 82.2 & 85.5 & 87.9 & 88.1 \\
\hline $35(60)$ & 80.9 & 83.6 & 86.5 & 88.1 & 88.9 \\
\hline $36(61)$ & 79.7 & 87.0 & 87.9 & 90.5 & 92.9 \\
\hline $37(120)$ & 86.2 & 87.6 & 90.1 & 91.2 & 94.5 \\
\hline $38(68)$ & 82.6 & 89.1 & 89.8 & 91.5 & 94.9 \\
\hline $39(45)$ & 87.5 & 89.6 & 91.7 & 92.0 & 95 \\
\hline $40(35)$ & 87.4 & 89.3 & 89.9 & 91.4 & 94.3 \\
\hline
\end{tabular}

Table 4. FL percentile chart for various gestational age

\begin{tabular}{|l|l|l|l|l|l|}
\hline WEEKS & \multicolumn{1}{|c|}{ 5TH } & \multicolumn{1}{|c|}{ 25TH } & \multicolumn{1}{|c|}{ 50TH } & \multicolumn{1}{|c|}{ 75TH } & 95TH \\
\hline 30 & 55.6 & 55.6 & 56.8 & 56.9 & \\
\hline 31 & 58.7 & 60.9 & 61.1 & 61.9 & 62.5 \\
\hline 32 & 58.3 & 60.9 & 61.5 & 62.9 & 65.2 \\
\hline 33 & 61.7 & 63.4 & 65.3 & 66.8 & 69 \\
\hline 34 & 61.3 & 63.2 & 64.3 & 67 & 68.2 \\
\hline 35 & 63.8 & 65.4 & 67.3 & 69.3 & 70.7 \\
\hline 36 & 65.2 & 68.3 & 69.9 & 70.8 & 73.9 \\
\hline 37 & 68.7 & 70.0 & 71.8 & 72.6 & 76.1 \\
\hline 38 & 67.4 & 70.8 & 72.3 & 73.6 & 75.7 \\
\hline 39 & 69.6 & 70.2 & 73.8 & 74.5 & 78 \\
\hline 40 & 69.4 & 72 & 73.2 & 76.8 & 77.4 \\
\hline
\end{tabular}

Table 5. AC percentile chart for various gestational age

\begin{tabular}{|l|l|l|l|l|l|}
\hline WEEKS & \multicolumn{1}{|c|}{ 5TH } & \multicolumn{1}{|c|}{ 25TH } & 50TH & 75TH & \multicolumn{1}{|c|}{ 95TH } \\
\hline 30 & 253 & 253 & 263.6 & 272.4 & \\
\hline 31 & 250.1 & 264.2 & 274.4 & 282 & 294.7 \\
\hline 32 & 264.2 & 277.4 & 282.1 & 296.4 & 298.1 \\
\hline 33 & 256 & 261.8 & 283 & 293.2 & 315 \\
\hline 34 & 280.6 & 281.8 & 300.4 & 306.5 & 316.1 \\
\hline 35 & 293.7 & 297.6 & 300.3 & 308.8 & 316 \\
\hline 36 & 299.4 & 301.9 & 313.6 & 320.4 & 328.5 \\
\hline 37 & 296 & 309.6 & 320.7 & 331.5 & 337.7 \\
\hline 38 & 307.4 & 317 & 328.4 & 337.6 & 353.8 \\
\hline 39 & 313.5 & 319.1 & 325.9 & 329.9 & 346 \\
\hline 40 & 315.8 & 321.1 & 323 & 330.7 & 331.4 \\
\hline
\end{tabular}




\section{DISCUSSION}

Assessment of fetal growth is an important aspect of antenatal care. Low birth weight fetuses are at higher risk for adverse perinatal outcome that are potentially avoidable. $(4,5)$ Assessment of growth in third trimester are usually based on gestational age specific growth curves. ISUOG practice guidelines defined appropriate for gestational age (AGA) fetus as those having EFW and AC between 10th and 90th percentile, small for gestational age (SGA) fetus as those having EFW and AC below 10th percentile and large for gestational age as those having EFW and AC above 90th percentile in growth curves. ${ }^{(2)}$ Many of the fetal growth chart that are in use are based on studies from high income countries. A study conducted by WHO demonstrated that apart from variables like maternal, age, height, parity, fetal sex, country its self also had significant effect of estimated fetal weight, probably related to ethnic and geographic variation. ${ }^{(3)}$ In this study we tried to assess fetal biometry and estimated fetal weight during third trimester ultrasound of term, appropriate birth weight neonates.

The median weeks of delivery and birth weight in our study is comparable to the multinational study done by $\mathrm{WHO}^{(3)}$ The median birth age is also comparable to the average birth weight reported in the Nation demographic and health survey 2011 . $^{(6)}$

A reference range for Nepalese population for fetal biometry has been generated from median value of biometry and estimated fetal weight calculated by Hadlock 2 formula. This can act as a reference for larger population-based study.

In our study the median percentile for expected fetal weight by WHO chart was about 30 and about $4 \%$ expected fetal weight was below 5th percentile according to $\mathrm{WHO}$ growth chart. This might be due to country related and maternal factors that influence the fetal growth. However, our study population represents urban population in mid to high socioeconomic status and also the median age at pregnancy is higher in our patients, thus population biometry and expected fetal weight might be different than that predicted by our study.

Our study had certain limitations. The study sample was small and study population was limited to a corporate hospital, so our study population was urban resident in mid to high socioeconomic status, and thus the data might not be true representative of the population.

In conclusion we generated a reference value for fetal biometry and expected fetal weight for Nepalese population. Expected fetal range in Nepalese population was smaller than that predicted by WHO chart. A larger populationbased study of fetal biometry and generation of country specific fetal growth curve is needed to correctly evaluated fetal growth.

\section{REFERENCES}

1. Gardosi J, Francis A, Turner S, et al. Customized growth charts: rationale, validation and clinical benefits. Am J Obstet Gynecol 2018; 218(2): S609-18.

2. Salomon LJ, Alfirevic Z, Da Silva Costa F, et al. ISUOG Practice Guidelines: ultrasound assessment of fetal biometry and growth. Ultrasound Obstet Gynecol 2019; 53(6): 715-23.

3. Kiserud T, Benachi A, Hecher K, et al. The World Health Organization fetal growth charts: concept, findings, interpretation, and application. Am J Obstet Gynecol 2018; 218(2): S619-29.

4. Williams M, Turner S, Butler E, et al. Fetal growth surveillance Current guidelines, practices and challenges. Ultrasound 2018 ; 26(2): 69-79.

5. Erkamp JS, Jaddoe VWV, Mulders AGMGJ, et al. Customized versus population birth weight charts for identification of newborns at risk of long-term adverse cardio-metabolic and respiratory outcomes: a population-based prospective cohort study. BMC Med 2019; 17(1): 186.

6. Khanal V, Sauer K, Karkee R, et al. Factors associated with small size at birth in Nepal: further analysis of Nepal Demographic and Health Survey 2011. BMC Pregnancy Childbirth 2014; 14(1): 32. 